Sebastián Genero, ${ }^{1}$

Eugenia M. Flores, ${ }^{2}$

Cristian E. Talavera, ${ }^{2}$

Ignacio Riveros, ${ }^{2}$

María E. Cañete, ${ }^{2}$

Tomás Osorio ${ }^{2}$

${ }^{1}$ Médico, Magister en Epidemiología aplicada a la Salud Pública, Especialista en Docencia Universitaria y en Clínica Médica. Prof.

Titular de la Cátedra de

Atención Primaria de la

Salud, Epidemiología e

Informática II, Facultad

de Medicina, Universidad

Nacional del Nordeste,

Jefe del Departamento de

Educación Permanente e

Investigación y Coordinador de la Residencia de

Epidemiología, del

Hospital Julio C. Perrando,

Resistencia Chaco

2Estudiante de medicina,

Ayudante alumno de la

Cátedra de Atención Primaria

de la Salud, Epidemiología

e Informática II, Facultad

de Medicina, Universidad

Nacional del Nordeste

Trabajo recibido: 02 de agosto de 2018. Aprobado: 24 de setiembre de 2018.

\section{TENDENCIA, DISTRIBUCIÓN GEOGRÁFICA Y DESIGUALDADES DE LA MORTALIDAD POR TUBERCULOSIS EN LA PROVINCIA DE CHACO} DURANTE EL PERIODO 2000-2016.

TENDENCY, GEOGRAPHICAL DISTRIBUTION AND INEQUALITY IN MORTALITY DUE TO TUBERCULOSIS IN THE PROVINCE OF CHACO DURING THE PERIOD 2000-2016.

TENDÊNCIA, DISTRIBUIÇÃO GEOGRÁFICA E DESIGUALDADES DE MORTALIDADE POR TUBERCULOSE NA PROVIINCIA DE CHACO DURANTE O PERIOODO 2000-2016

\section{Resumen}

Objetivos: describir la tendencia y distribución geográfica de la mortalidad por tuberculosis (TBC)en la provincia del Chaco y analizar su relación con las condiciones de vida de la población. Métodos: diseño ecológico. Se calcularon tasas de mortalidad generales y específicas, brutas y ajustadas a la población mundial, se analizaron tendencias del período mediante regresión segmentaria y desigualdades mediante curva de concentración y regresión lineal. Resultados: durante el período de estudio se produjeron 978 muertes por TBC, la tasa de mortalidad del período resultó en 5,4 por cada 100000 habitantes. El riesgo de muerte fue diferente según sexo, edad y departamento de residencia. La mortalidad descendió $-3.6 \%$ por año (IC $95 \%-4.9 ;-2.2 ; \mathrm{p}<0,05$ ) y el 
Índice de concentración resultó en -0,33. Conclusión: La mortalidad por TBC se encuentra descendiendo, pero se evidenciaron inequidades que deben ser tenidas en cuenta en la planificación de las políticas de Salud Pública.

Palabras Clave: Mycobacterium tuberculosis, Mortalidad, Disparidades en el Estado de Salud.

\section{Abstract}

Objectives: To describe the tendency and geographical distribution of mortality due to tuberculosis (TB) in the province of Chaco, and analyze the life condotions of the population. Method: ecological design. General and specific mortality rates were calculated, in the rough and adjusted to world population; the tendencies of the period were analyzed through segmented regression and inequalities through concentration curve and linear regression. Results: during the period studied there were 978 deaths because of TB, mortality rate resulted in 5.4 for every 100,000 inhabitants. Risk of death was different according to sex, age and department of residence. Mortality decreased $-3.6 \%$ per year (IC $95 \%$ $-4.9 ;-2.2 ; \mathrm{p}<0.05)$ and concentration index resulted in -0.33 . Conclusion: Mortality due to TB is decreasing; however, there are inequalities that must be taken into account when planning Public Health policies.

Key Words: TB Mycobacterium, Mortality, Inequalities in Health Conditions.

\section{Resumo}

Objetivos: descrever a tendência e distribuição geográfica da mortalidade por tuberculose (TBC) na província de El Chaco e analisar sua relação com as condições de vida da população. Métodos: design ecológico. Foram calculadas taxas de mortalidade gerais e específicas, brutas e ajustadas para a população mundial, as tendências dos períodos foram analisadas por regressão segmentar e as desigualdades por curva de concentração e regressão linear. Resultados: Durante o período do estudo houve 978 mortes por tuberculose, a taxa de mortalidade no período foi de 5,4 para cada 100.000 habitantes. O risco de morte foi diferente de acordo com sexo, idade e distrito de residência. A mortalidade diminuiu $-3,6 \%$ ao ano (IC 95\% - -4,9, -2,2, p <0,05) e o Índice de concentração foi de $-0,33$. Conclusão: A mortalidade por tuberculose está diminuindo, mas houveram iniquidades que devem ser levadas em consideração no planejamento das políticas de saúde pública.

Palavras chave: Mycobacterium tuberculosis, Mortalidade, Disparidades no Estado de Saúde.

\section{Introducción}

La tuberculosis (TBC) es una enfermedad infectocontagiosa, producida por el Mycobacterium tuberculosis que afecta más frecuentemente al pulmón, aunque puede afectar otros órganos. A pesar de que el diagnóstico precoz y el tratamiento adecuado son capaces de lograr la curación en la mayoría de los pacientes, la falta de detección de casos, el abandono del tratamiento, la aparición de resistencia a los fármacos antituberculosos tradicionales, la endemia del VIH, las malas condiciones socioeconómicas, la desnutrición y el alcoholismo $(1,2,3,4,5)$, entre otros factores individuales y sociales, generan que la TBC, en la actualidad, siga siendo un importante problema pública. Según la Organización Mundial de la Salud, la TBC, es la novena causa mundial de muerte y la primera por enfermedades infecciosas producidas por un solo microorganismo.

En 2016 la cifra estimada de muertes por TBC fue de 1,3 millones en personas VIHnegativas, y de 374000 en personas VIH-positivas.(6)La provincia del Chaco, es una de 
las jurisdicciones de la República Argentina, con indicadores sociales más desfavorables, y la tercera con la tasa de mortalidad más alta por TBC.(7) Por otra parte, se sabe que las malas condiciones de vida de las poblaciones determinan diferencias en la expresión de la carga de enfermedad y muerte y producen desigualdades que resultan inaceptables (8), $\mathrm{y}$, por ello, el análisis de la distribución desigual de los eventos de salud en general, y el de la TBC en particular, en relación a los determinantes sociales, resulta de interés para poder identificar áreas donde las acciones deben priorizarse. Previamente, en nuestro país se han identificado desigualdades sociales tanto en la mortalidad como en la incidencia de esta enfermedad. $(7,9)$

Este trabajo tuvo como objetivo describir la tendencia y distribución geográfica de la mortalidad por esta patología en la provincia del Chaco, durante el periodo 2000-2016, y analizar su relación con las condiciones de vida de la población.

\section{Materiales y Métodos}

Tipo de estudio: Se realizó un estudio ecológico de tipo mixto (series de tiempo y grupos múltiples).

Universo y muestra: el universo corresponde a las defunciones por tuberculosis, producidas en residentes de la provincia del Chaco. En este trabajo se utilizan todos los casos registrados en la fuente de datos durante el período de estudio, con los códigos A15-A19 y B90 de la décima revisión de la Clasificación Internacional de Enfermedades. Unidad de análisis: para el análisis de series de tiempo se consideraron todas las defunciones por tuberculosis registradas por certificado de defunción, por año, en el periodo 2000 a 2016 en la provincia del Chaco y, para la comparación de los grupos, se consideró como conglomerado, a cada uno de los departamentos de la provincia del Chaco.

Fuente de datos: se utilizaron las bases de datos de la Dirección Nacional de Estadísticase Información en Salud, del Ministerio de Salud de la Nación, de los años 2000 a 2016 para la obtención de las muertes, y el censo de población y viviendas de la República Argentina y proyecciones realizadas por el INDEC, para la estimación de los denominadores poblacionales y, para la determinación de las necesidades básicas insatisfechas (NBI) a nivel departamental.

Análisis estadístico: Se calcularon la proporción de muertes según edad ytasas de mortalidad específicas por edad, utilizando las siguientes categorías: 0 a 4, 5 a 14, 15 a 24, 25 a 44, 45 a 64, 65 a 74 y 75 años y más. Estas categorías de edad fueron seleccionadas para mantener la uniformidad con los registros provinciales de las estadísticas de mortalidad, y con el propósito de posibilitar comparaciones con las mismas en el futuro. También se calcularon la tasa de mortalidad por tuberculosis, general y por sexo, por año del periodo, y tasas estandarizadas por edad, ajustadas a la población mundial, de la Organización Mundial de la Salud. Las tasas de mortalidad general y por sexo fueron suavizadas mediante su cálculo en períodos trienales. Para el análisis de la tendencia del período y para la evaluación de la relación entre la tasa de mortalidad y las necesidades básicas insatisfechas de la población, se utilizó regresión lineal simple, mediante la ecuación:

$$
\mathrm{y}=\mathrm{B} 0+\mathrm{B} 1{ }^{*} \mathrm{x}
$$

Donde y representa la tasa de mortalidad, B0 la constante, B1 el cambio medio de la tasa de mortalidad por cada cambio de $\mathrm{x}$, siendo $\mathrm{x}$, el año que se considere.

Para el análisis de tendencias de las tasas general, por sexo y por grupos de edad de mortalidad por TBC, se aplicó el método de regresión segmentaria, donde la variable dependiente fue la tasa de mortalidad por TBC y, la independiente, cada año del período, 
en base a la distribución de Poisson, utilizando el software Joinpoint Regression Program, Version 4.6.0.0 de abril de 2018, del Statistical Research and Applications Branch, National Cancer Institute de los Estados Unidos. Se consideraron significativos valores de $\mathrm{p}$ menores a 0,05 .

El cálculo de las tasas de mortalidad por TBC para el período 2000-2016, por cada departamento, se estimó mediante:

P (d) 2008

$$
\mathrm{T}(\text { departamento })=\overline{\mathrm{x}} \mathrm{M}(\mathrm{d})
$$

Donde $\overline{\mathrm{x}} \mathrm{M}(\mathrm{d})$ representa la media de muertes por TBC en residentes en cada departamento de la provincia del Chaco, en el período 2000-2016 y P (d) 2008 la población del año 2008, (mitad del período) de cada departamento. Se estimó el riesgo relativo (RR) entre cada departamento y dos puntos de referencia: el departamento de menor tasa de mortalidad y la tasa de mortalidad por TBC, de la provincia, de todo el período. Para ilustrar la distribución geográfica se construyó un mapa temático, mediante el software ArcGIS versión 1.4, indicándose con color verde los departamentos con tasa de mortalidad igual o por debajo del primer tercil, con amarillo, los departamentos con tasas de mortalidad igual o por debajo del segundo tercil y con rojo aquellos con tasas de mortalidad por encima del segundo tercil.

Teniendo en cuenta que los eventos de salud no se distribuyen en forma aleatoria, y se encuentran determinados socialmente(10), se realizó un análisis de la relación entre condiciones socioeconómicas y mortalidad por $\mathrm{TBC}$, utilizando como indicador de las condiciones socioeconómicas de la población, el índice NBI. Este índice considera diferentes dimensiones que definen niveles críticos de privación social y fue seleccionado para este trabajo, dado que es elaborado mediante censos de la población, presentando por ello representatividad geográfica y se encuentra disponible con el nivel de desagregación requerida. Se siguió el siguiente procedimiento: se dividieron los departamentos de la provincia según quintiles en base a las tasas de mortalidad ordenadas de mayor a menor. Por cada departamento se estimó la media del porcentaje de población con necesidades básicas insatisfechas, en base a los censos 2001 y 2010, y a cada quintil se le adjudicó la mediana de las medias obtenidas. Se calcularon RR considerando las tasas de mortalidad de cada quintil, con el quintil de menor tasa de mortalidad. Se aplicó, como se mencionó previamente, regresión lineal para estimar el cambio de la tasa de mortalidad por cada punto de cambio en el porcentaje de NBI, tomando como unidad de análisis cada quintil. También se utilizó la curva y el coeficiente de concentración, utilizando como indicador socioeconómico el porcentaje de población con NBI por cada departamento y como indicador de salud, la tasa de mortalidad por TBC. Estos últimos fueron analizados mediante EPI DAT, versión 4.2.

\section{Resultados}

Tendencia del período según sexo.

Durante el período de estudio se produjeron 978 muertes por tuberculosis, a razón de una media de $57 \pm 11$ muertes por año. La tasa de mortalidad del período resultó en 5,4 casos por cada 100000 habitantes. El riesgo de muerte fue de 6,5 por 100000 varones y 4,3 por 100000 mujeres, lo que resulta en un riesgo relativo de 1,5. En la Fig. $\mathrm{N}^{\circ} 1$ se presenta la tendencia de la tasa de muerte por año, según sexo. 


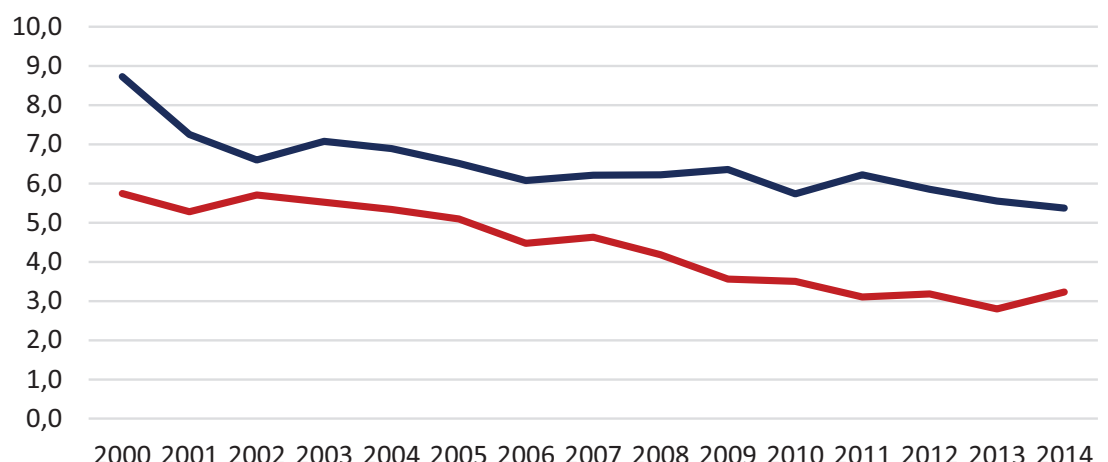

200020012002200320042005200620072008200920102011201220132014

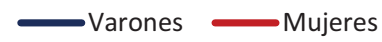

Fig. №1 Tasa de mortalidad por 100000 habitantes, por TBC, según sexo, provincia del Chaco, 2000-2016

Fuente: Dirección Nacional de Estadísticas e Información en Salud, Ministerio de Salud de la Nación, y proyecciones de población del INDEC. Elaboración propia.

La tendencia reconoció un descenso del orden de 0,2 puntos de la tasa por año $(\mathrm{R} 2=0,66)$ según estimación lineal y de $0,19\left(\mathrm{R}^{2}=0,94\right)$ para la estimación lineal de la tasa suavizada por trienio. En términos porcentuales, la tasa bruta general descendió a razón de $-3.6 \%$ por año(IC $95 \%-4.9 ;-2.2 ; \mathrm{p}<0,05)$ y la ajustada a la edad de la población mundial de la OMS, $-4,4 \%$ por año (IC95\% $-5,8 ;-3,0 ; \mathrm{p}<0,05)$ El descenso en la mortalidad fue diferente según sexo, siendo más pronunciado en las mujeres. En varones el descenso porcentual de la tasa anual fue del orden de -3.0 (IC95\%-5.0;-1.0; $<<0,05)$ y en las mujeres de -4.4 (IC $95 \%-6.4 ;-2.3 ; \mathrm{p}<0,05)$.

Proporción de muertes por edad, y tasa de mortalidad según sexo y edad.

Aunque la mayor cantidad de muertes se concentró en las personas de 45 a 64 años, el mayor riesgo se produjo en las de 75 años y más. (Fig. No 2 y N³) A su vez, en todos los grupos, excepto en los menores de 14 años, el riesgo fue mayor en varones que en mujeres. (Fig. No3)
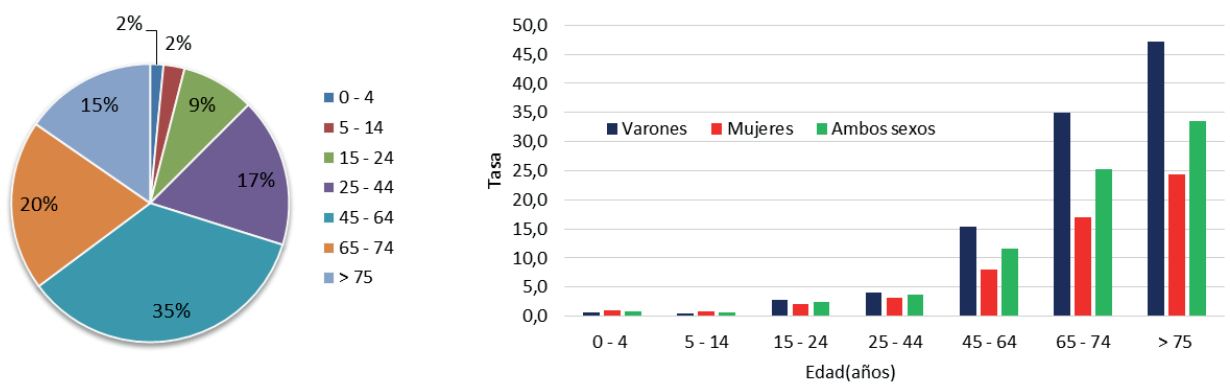

Fig. № 2 Proporción de muertes por TBC, según edad, provincia del Chaco, 2000-2016, n=975

Fig. № 3 Tasa de mortalidad por TBC, por 100000 habitantes, según sexoy edad, provincia del Chaco, 2000-2016

Fuente: Dirección Nacional de Estadísticas e Información en Salud, Ministerio de Salud de la Nación, y proyecciones de población del INDECElaboración propia.

Tendencia de la tasa de muerte según edad

En la población de 0 a 4 años existió un mínimo de 1 muerte por año y un máximo de 4 (en los años 2002 y 2003) y desde el año 2010 no se verificaron muertes en este estrato de edad.

En la población de 5 a 14 años, también se verificó un mínimo de 1 muerte por año y un 
máximo de 4 (en el año 2002) y desde el año 2010 no se registraron muertes en este grupo de edad, con excepción de los años 2012 (2 muertes) y 2016 (1 muerte). La existencia de años durante el período con ninguna muerte en estas edades no permitió realizar el análisis de tendencia.

La exploración de la distribución de la mortalidad en las personas de 15 a 24 años, no presentó patrón lineal y por ello las estimaciones proporcionaron un coeficiente de determinación cercano a cero, incluso cuando se estimó la ecuación de regresión con tasas suavizadas utilizando periodos trienales. $(\mathrm{R} 2=0,03)$ y por ello no se calculó el cambio porcentual anual. Sin embargo, resulta importante mencionar que en los primeros cinco años del período la tasa de mortalidad en este grupo de edad fue de 2,07 por cien mil y en los últimos 5 años fue de 2,67 por cien mil, lo que sugiere una tendencia al aumento, lo que no ha ocurrido en ninguno de los demás estratos de edad estudiados.

En el resto de los grupos de edad, donde fue posible realizar el análisis de tendencia, se observó decrementos en todos ellos, siendo las personas de 75 años y más, las que evidenciaron una disminución de mayor intensidad en el riesgo de muerte por esta causa. (Tabla $\left.\mathrm{N}^{\mathrm{o}} 1\right)$

Tabla $\mathbf{N}^{\circ} 1$ : Tendencia de mortalidad por TBC, según edad, provincia del Chaco , 20002016

\begin{tabular}{l|c|c|c|}
\cline { 2 - 3 } \multirow{2}{*}{ Edad (años) } & \multicolumn{2}{|c|}{ Años } & \\
\cline { 2 - 3 } & 2000 & 2016 & $\begin{array}{c}\text { \% de cambio anual (IC } \\
95 \%)\end{array}$ \\
\hline 0 a 4 & 2,5 & 0,0 & No se evalúa \\
\hline 5 a 14 & 1,3 & 0,5 & No se evalúa \\
\hline 15 a 24 & 3,7 & 5,3 & No se evalúa \\
\hline 25 a 44 & 5,8 & 4,4 & $-1.8^{*}(-4.8 ; 1.4)$ \\
\hline 45 a 64 & 16,8 & 5,5 & $-6.0^{*}(-8.7 ;-3.2)$ \\
\hline 65 a 74 & 40,8 & 27,9 & $-2.6^{*}(-4.7 ;-0.5)$ \\
\hline 75 y mas & 79,7 & 8,6 & $-6.4^{*}(-9.9 ;-2.8)$ \\
\hline $\begin{array}{l}\text { Todas las } \\
\text { edades }\end{array}$ & 8,5 & 4,8 & $-3.6^{*}(-4.9 ;-2.2)$ \\
\hline
\end{tabular}
${ }^{*} p<0,05$

Fuente: Dirección Nacional de Estadísticas e Información en Salud, Ministerio de Salud de la Nación, y proyecciones de población del INDEC. Elaboración propia.

Tabla N²: Tasa de mortalidad por TBC según departamento y riesgo relativo respecto de la provincia y de la menor tasa departamental del período, provincia del Chaco, 2000-2016

\begin{tabular}{l|ccc}
\multicolumn{1}{c|}{ Departamento } & $\begin{array}{c}\text { Tasa por } \\
100000 \mathrm{~h}\end{array}$ & $\begin{array}{c}\text { RR respecto } \\
\text { de la } \\
\text { provincia }\end{array}$ & $\begin{array}{c}\text { RR respecto } \\
\text { de la menor } \\
\text { tasa }\end{array}$ \\
General Güemes & 25,0 & 4,6 & 21,3 \\
O'Higgins & 8,7 & 1,6 & 7,4 \\
General Belgrano & 8,2 & 1,5 & 7,0 \\
Libertador General San Martín & 7,6 & 1,4 & 6,5 \\
25 de Mayo & 6,3 & 1,2 & 5,4
\end{tabular}


Quitilipi

Sargento Cabral

San Lorenzo

Bermejo

Mayor Luis J. Fontana

Independencia

2 de Abril

General Donovan

Comandante Fernández

Maipú

9 de Julio

Presidencia de la Plaza

San Fernando

Almirante Brown

Tapenagá

Chacabuco

Libertad

Fray Justo Santa María de Oro

12 de Octubre

$1^{\circ}$ de Mayo

\begin{tabular}{|c|c|c|}
\hline 6,2 & 1,1 & 5,3 \\
\hline 5,9 & 1,1 & 5,0 \\
\hline 5,2 & 1,0 & 4,4 \\
\hline 5,0 & 0,9 & 4,2 \\
\hline 5,0 & 0,9 & 4,2 \\
\hline 4,9 & 0,9 & 4,2 \\
\hline 4,7 & 0,9 & 4,0 \\
\hline 4,1 & 0,8 & 3,5 \\
\hline 4,1 & 0,8 & 3,5 \\
\hline 3,9 & 0,7 & 3,3 \\
\hline 3,4 & 0,6 & 2,9 \\
\hline 3,2 & 0,6 & 2,7 \\
\hline 3,1 & 0,6 & 2,7 \\
\hline 2,9 & 0,5 & 2,5 \\
\hline 2,6 & 0,5 & 2,3 \\
\hline 2,2 & 0,4 & 1,8 \\
\hline 2,0 & 0,4 & 1,7 \\
\hline 1,8 & 0,3 & 1,5 \\
\hline 1,3 & 0,2 & 1,1 \\
\hline 1,2 & 0,2 & 1,0 \\
\hline
\end{tabular}

Fuente: Dirección Nacional de Estadísticas e Información en Salud, Ministerio de Salud de la Nación, y proyecciones de población del INDEC. Elaboración propia.

De los 25 departamentos de la provincia, en 7 de ellos se evidenciaron tasas mayores de mortalidad respecto de la tasa provincial y del departamento con menor riesgo de muerte por esta causa. Los residentes del departamento General Güemes concentraron el 30,1\% del total de las muertes por TBC y presentaron 4,6 más riesgo de muerte respecto del riesgo de la provincia y 21,3 veces más riesgo respecto de los habitantes del departamento $1^{\circ}$ de Mayo. Estas diferencias se exponen en la Tabla $\mathrm{N}^{\circ} 2$.

En el mapa $\mathrm{N}^{\circ} 1$ se presenta la distribución del riesgo de muerte por departamento, según terciles de las tasas de mortalidad, y se evidencia que las regiones central y norte de la provincia presentaron mayor riesgo de mortalidad. 


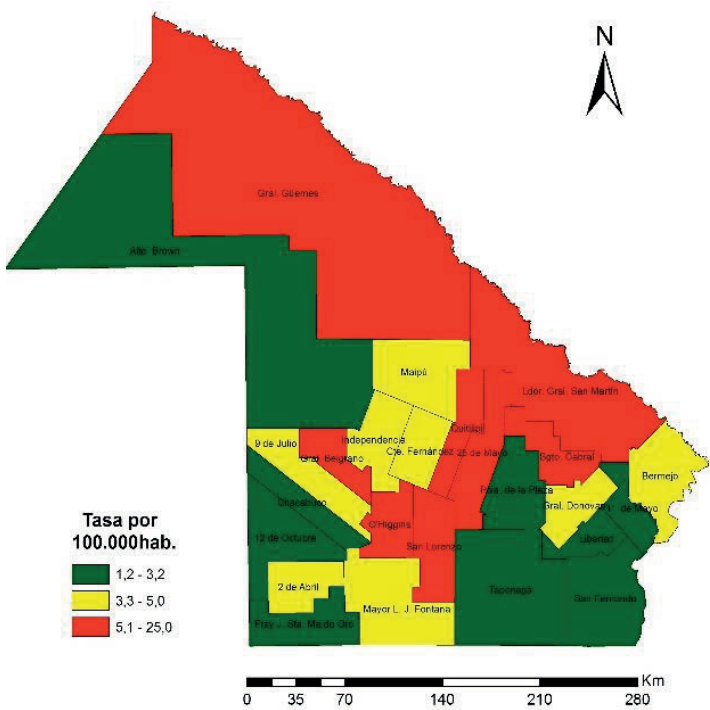

Mapa $\mathrm{N}^{\mathrm{0}} 1$ Tasa de mortalidad de TBC por 100000 habitantes, por departamento, provincia del Chaco, 2000-2016

Fuente: Dirección Nacional de Estadísticas e Información en Salud, Ministerio de Salud de la Nación, y proyecciones de población del INDEC. Elaboración propia.

\section{Desigualdades sociales en la mortalidad por TBC}

Al estimar la desigualdad en la distribución de la tasa de mortalidad según el porcentaje de población con necesidades básicas insatisfechas por departamento, la curva de concentración se situó por encima de la diagonal de igualdad y reflejó el $50 \%$ de la población que reside en los departamentos con peores condiciones socioeconómicas concentró aproximadamente el $70 \%$ de los casos (Índice de concentración= - -0,33)

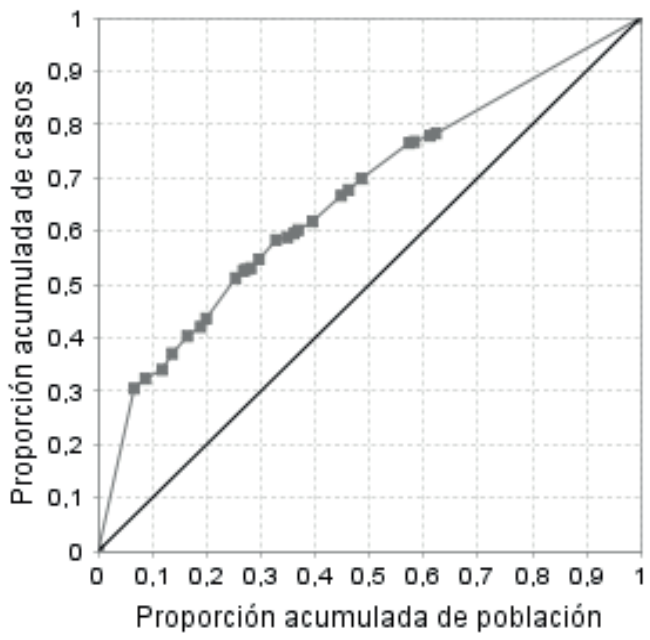

Fig. Nº Curva de concentración de la tasa de mortalidad por TBC según departamentos con necesidades básicas insatisfechas, provincia del

Fuente: Dirección Nacional de Estadísticas e Información en Salud, Ministerio de Salud de la Nación, y proyecciones de población del INDEC. Elaboración propia.

El riesgo de muerte por TBC, según departamentos ordenados por quintiles en base al porcentaje de población con necesidades básicas insatisfechas, presentó un gradiente de 
riesgo por el cual los habitantes de los departamentos con condiciones socioeconómicas más desfavorables presentaron 4,2 veces mayor riesgo de muerte respecto de los habitantes de los departamentos con mejores condiciones. (Tabla No3)

Tabla N³: Mortalidad por TBC según quintiles de departamentos con necesidades básicas insatisfechas, provincia del Chaco, 2000-2016

\begin{tabular}{l|cccccc}
\hline Quintil & $\begin{array}{c}\text { Muertes } \\
\text { del } \\
\text { período }\end{array}$ & $\begin{array}{c}\text { Media por } \\
\text { año }\end{array}$ & $\begin{array}{c}\text { Población } \\
2008\end{array}$ & $\begin{array}{c}\text { Mediana } \\
\text { NBI }\end{array}$ & $\begin{array}{c}\text { Tasa por } \\
100000 \mathrm{~h}\end{array}$ & RR \\
1 & 391 & 23 & 172978 & 34,8 & 13,3 & 4,2 \\
2 & 122 & 7 & 118264 & 30,2 & 6,1 & 1,9 \\
3 & 65 & 4 & 88555 & 27,6 & 4,3 & 1,4 \\
4 & 100 & 6 & 130531 & 23,2 & 4,5 & 1,4 \\
5 & 293 & 17 & 541850 & 19,5 & 3,2 & 1,0
\end{tabular}

Fuente: Dirección Nacional de Estadísticas e Información en Salud, Ministerio de Salud de la Nación, y proyecciones de población del INDEC. Elaboración propia.

\section{Discusión}

En nuestro trabajo se identificó una tendencia al descenso de la tasa de mortalidad por TBC, resultados similares han sido reportados en todas las regiones de la Organización Mundial de la Salud, para los años 2000-2016, (6) en las cuales el porcentaje de cambio anual de la tasa de mortalidad estandarizada a la edad de la población mundial, osciló entre $-2,2 \%$,en los países del Este del Mediterráneo, a - $6 \%$ en Europa. Este fue, según nuestro conocimiento, el único estudio publicado que, utilizó el mismo método de estandarización de las tasas y el mismo período de tiempo que nuestro trabajo. La magnitud del descenso observada en nuestro trabajo también fue similar a la reportada en otros estudios $(6,11-15)$, sin embargo, en ellos se utilizaron otras poblaciones estándar o períodos de tiempos diferentes al nuestro, lo que resulta en una limitación para realizar comparaciones.

Tal como ocurre en otras publicaciones $(7,9,13,16,17)$, en nuestro trabajo se evidenció una diferencia en el riesgo de muerte por TBC, de tal manera que las personas de sexo masculino presentaron mayor probabilidad de morir por esta enfermedad respecto de las del sexo femenino. No sólo resultó diferente el riesgo de muerte, sino también, el cambio porcentual anual de la tendencia según sexo, la cual, en nuestro trabajo, presentó un descenso mayor en la población del sexo femenino, en todo el período analizado. En este sentido, en Europa, para el periodo 2000-2010, el cambio porcentual anual de la tasa ajustada a la edad del sexo masculino fue de $-7 \%$ y del sexo femenino del orden de $-8,1 \%(13)$, mientras queun estudio realizado en el estado de Paraná (Brasil), se observó, para el período 1998-2010, un mayor decremento en la mortalidad del sexo masculino, y un aumento para el periodo 2010-2012(18).

Los resultados de nuestro trabajo son consistentes con lo comunicado previamente en relación a la distribución del riesgo de muerte según edad, el cual presenta una asociación directa con la edad. En nuestro país, se ha determinado el aumento de la tasa de mortalidad del orden de 5,6\%, por año de vida, en mujeres y 7,3\%, por año de vida, en varones(7). También se observó que la tasa de mortalidad específica por edad, aumenta marcadamente, a partir de los 30 años de edad y es máxima entre los 55 a 74 años.(9) En nuestro trabajo, si bien se evidencia un gradiente de riesgo conforme aumenta la edad, la tasa de mortalidad 
específica por edad aumentó luego de los 45 años y fue máxima en las personas de 75 años y más de edad. Esta diferencia podría deberse a que las categorías de edad estudiadas no se corresponden exactamente con las del trabajo de referencia, aunque también podrían atribuirse a diferencias en los factores de riesgo de la población, por otra parte, resultados similares a nuestro trabajo fueron comunicados para nuestro país en el período 1990-2008. (17)

Aunque han sido ampliamente descriptas por diversos autores las desigualdades sociales en la incidencia de $\operatorname{TBC}(2,4,5,19-23)$, las desigualdades en la mortalidad no han sido evaluadas con la misma profundidad. En nuestro trabajo se evidenció un gradiente de riesgo de muerte por $\mathrm{TBC}$ asociado a malas condiciones socioeconómicas, como previamente se había observado en un estudio realizado en nuestro país con datos referidos al bienio 2014-2015, en el cual el riesgo relativo entre la provincia con mayor tasa de mortalidadpor TBC (Jujuy, 6,9 por cien mil habitantes) y la menor menor (La Pampa, 0,25 por cien mil habitantes), resultó en 27,6. Estas diferencias observadas a nivel provincial, también se presentaron cuando se analizó la mortalidad tomando como unidad de análisis los departamentos del país, donde se encontró la tasa mínima en el departamento Río Cuarto, de la provincia de Córdoba ( 0,19 por cien mil habitantes) y la tasa máxima en el departamento General Güemes, de nuestra provincia (26,8 por cien mil habitantes), es decir, un $\mathrm{RR}=141$. En este estudio de referencia nacional(7), el índice de concentración del nivel departamental resultó en -0,24, en tanto que en nuestro trabajo fue de -0,33 denotando, en nuestro trabajo, una mayor concentración de la tasa de mortalidad en los departamentos con mayor porcentaje de necesidades básicas insatisfechas. Otros trabajos publicados han referido asociación entre condiciones socioeconómicas y mortalidad por $\operatorname{TBC}(2,24)$ pero con indicadores diferentes al utilizado en nuestro trabajo.

Algunas limitaciones deben ser tenidas en cuenta en la lectura de nuestro trabajo: hemos utilizado registros de mortalidad que se confeccionan en base a certificados de defunción. Estos certificados pueden presentar diferencias en cuanto a su calidad, por motivos inherentes a los servicios de salud, y de esta manera, la definición de la causa de muerte, pudo haber estado influida por la capacidad diagnóstica del lugar de ocurrencia del evento, así como pudieron haberse producido errores de clasificación, teniendo en cuenta la presencia o no de factores de riesgo de los fallecidos. Por otra parte, el uso de períodos de tiempos diferentes o el uso de poblaciones estándar diferentes a la nuestra limitó nuestra posibilidad de comparación con otros estudios realizados en el país y en el mundo.

\section{Conclusiónes}

La mortalidad por TBC se encuentra descendiendo en la provincia del Chaco, pero se evidenciaron inequidades que deben ser tenidas en cuenta en la planificación de las políticas de Salud Pública. Este trabajo, además de documentar la existencia de las mismas, provee de una línea de base para realizar el seguimiento de los resultados de su implementación en el mediano y largo plazo.

\section{Bibliografía}

1. Ministerio de Salud de la Nación, República Argentina. Guia para el equipo de salud. Tuberculosis. [Internet]. 2014 [citado 25 de mayo de 2018]. Disponible en: http:/www.msal.gob.ar/images/stories/bes/graficos/0000000049cnt-guia_de_diagnostico_tratamiento_y_prevencion_de_la_tuberculosis_2015.pdf

2. Nagavci BL, de Gelder R, Martikainen P, Deboosere P, Bopp M, Rychtaříková J, et al. Inequalities in tuberculosis mortality: long-term trends in 11 European countries. Int J Tuberc Lung Dis. 1 de mayo de 2016;20(5):574-81. 
3. Zerbini E, Greco A, Estrada S, Cisneros M, Colombo C, Beltrame S, et al. Risk factors associated with tuberculosis mortality un adults in six provinces of Argentina. Medicina (B Aires). 2017;77:267-73.

4. Yamamura M, Santos-Neto M, Santos RAN dos, Garcia MC da C, Nogueira J de A, Arcêncio RA. Epidemiological characteristics of cases of death from tuberculosis and vulnerable territories. Rev Lat Am Enfermagem. octubre de 2015;23(5):910-8.

5. Horne DJ, Hubbard R, Narita M, Exarchos A, Park DR, Goss CH. Factors associated with mortality in patients with tuberculosis. BMC Infect Dis. 27 de agosto de 2010;10:258.

6. World Health Organization. Global tuberculosis report 2017 [Internet]. Geneva; 2017 [citado 22 de mayo de 2018]. Disponible en: http://www.who.int/tb/publications/global_report/MainText_13Nov2017.pdf

7. Instituto Nacional de Enfermedades Respiratorias (INER) "Emilio Coni” Administración Nacional de Laboratorios e Institutos de Salud (ANLIS) "Carlos G. Malbrán” Ministerio de Salud, República Argentina. Mortalidad por tuberculosis en la República Argentina. Período 1980-2015.

8. Schneider MC, Castillo-Salgado C, Bacallao J, Loyola E, Mujica OJ, Vidaurre M. Métodos de medición de las desigualdades de salud. Rev Panam Salud Publica. 2002;18.

9. Instituto Nacional de Enfermedades Respiratorias (INER) "Emilio Coni”, Administración Nacional de Laboratorios e Institutos de Salud (ANLIS) "Carlos G. Malbrán”, Ministerio de Salud, República Argentina. Mortalidad por Tuberculosis en Argentina, 1980-2014 [Internet]. 2016 [citado 23 de mayo de 2018]. Disponible en:http://www.anlis.gov.ar/iner/wp-content/uploads/2016/04/BoletinActualizacionMortalidadTuberculosisDocTec0316.pdf

10. Starfield B. Equity and health: a perspective on nonrandom distribution of health in the population. Rev Panam Salud Pública [Internet]. diciembre de 2002 [citado 2 de agosto de 2018];12(6). Disponible en: http://ojps.aip.org/link/?apl/74/2268/ab

11. Traebert J, Ferrer GCN, Nazário NO, Schneider IJC, Silva RM da. Tendência temporal da morbidade e mortalidade por tuberculose no estado de Santa Catarina, Brasil, no período entre 2002 e 2009. J Bras Pneumol. diciembre de 2012;38(6):771-5.

12. Ochoa EG, Oliva GER, Gutiérrez SB, González AP, Pérez LA. Tuberculosis Mortality Trends in Cuba, 1998 to 2007. MEDICC Rev. 2009;11(1):6.

13. Al-Rahamneh MJ, Al-Rahamneh A, Guillén-Grima F, Arnedo-Pena A, AguinagaOntoso I. Mortality trends for tuberculosis in European Union countries, 20002010. Enfermedades Infecc Microbiol Clínica [Internet]. julio de 2017 [citado 22 de mayo de 2018]; Disponible en: http://linkinghub.elsevier.com/retrieve/pii/ S0213005X1730191X

14. Barnes RFW, Moore ML, Garfein RS, Brodine S, Strathdee SA, Rodwell TC. Trends in Mortality of Tuberculosis Patients in the United States: The Long-Term Perspective. Ann Epidemiol. octubre de 2011;21(10):791-5.

15. Trends in Tuberculosis Morbidity and Mortality [Internet]. American Lung Association Research and Health Education Epidemiology and Statistics Unit; 2013 abr. Disponible en: http://www.lung.org/assets/documents/research/tb-trend-report.pdf

16. Bierrenbach AL, Duarte EC, Gomes ABF, Souza M de FM de. Tendência da mortalidade por tuberculose no Brasil, 1980 a 2004. Rev Saúde Pública. septiembre de 2007;41(suppl 1):15-23.

17. Ministerio de Salud de la Nación, Boletín epidemiológico períodico, Situación de la tuberculosis [Internet]. Ministerio de Salud de la Nación, República Argentina; 2011 [citado 25 de mayo de 2018]. Disponible en: http://www.msal.gob.ar/saladesituacion/boletines_epidemiologia/pdfs/BEP46_TBC_int.pdf

18. Cecilio HPM, Santos A de L, Marcon SS, Latorre M do RD de O, Mathias TA de F, 
Rossi RM. Tendência da mortalidade por tuberculose no estado do Paraná, Brasil 1998 a 2012. Ciênc Saúde Coletiva. enero de 2018;23(1):241-8.

19. Cabrera PL, Victoria P, Andalia RR. Aproximación teórica a las desigualdades sociales en la tuberculosis como problema de salud. Rev Cuba Salud Pública. :15.

20. Alves Filho P, Pellegrini Filho A, Ribeiro PT, Toledo LM de, Romão AR, Novaes LCM. Desigualdades socioespaciais relacionadas à tuberculose no município de Itaboraí, Rio de Janeiro. Rev Bras Epidemiol. diciembre de 2017;20(4):559-72.

21. Munayco CV, Mújica OJ, León FX, Granado M del, Espinal MA. Social determinants and inequalities in tuberculosis incidence in Latin America and the Caribbean. Rev Panam Salud Pública. septiembre de 2015;38:177-85.

22. Bossio JC, Arias SJ, Fernández HR. Tuberculosis en Argentina: desigualdad social y de género. Salud Colect. noviembre de 2012;8:77-91.

23. Apolinário D, Ribeiro AI, Krainski E, Sousa P, Abranches M, Duarte R. Tuberculosis inequalities and socio-economic deprivation in Portugal. Int J Tuberc Lung Dis. 1 de julio de 2017;21(7):784-9.

24. Sánchez-Barriga JJ. Tendencias de mortalidad y riesgo de muerte por tuberculosis pulmonar en las 7 regiones socioeconómicas y los 32 estados de México, 20002009. Arch Bronconeumol. enero de 2015;51(1):16-23. 